\title{
The Research of Space Coordinate Transform Algorithm for Test Bench of Automobile Suspension Kinematics
}

\author{
Xiaohui Bai ${ }^{1, a}$, Bowei $\mathrm{Bi}^{1, \mathrm{~b}}$, Huang Yong ${ }^{1, \mathrm{c}}$ and Haiyang $\mathrm{Ou}^{1, \mathrm{~d}}$ \\ ${ }^{1}$ Transportation and Automotive Engineering Institute, Key Laboratory of Sichuan Province \\ Automobile Engineering, Xihua University, Chengdu, 610000, China

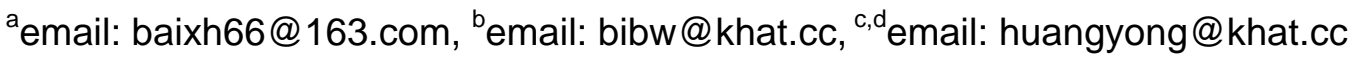

Keywords: Coordinate Transformation; Suspension Kinematics

\begin{abstract}
Suspension Kinematic is important feature for automobile. This paper focus on space coordinate transform algorithm of Suspension Kinematic parameters. New algorithm for the Suspension Kinematics test bench is based on theory of space vector and coordinate geometry transform. Relative coordinates of specific point is core measure content for algorithm. After comparing result of algorithm and ADAMS simulation, the accuracy and efficiency of algorithm is proved. Space Coordinate Transform algorithm can be used to the theoretical calculation of Suspension Kinematics feature, such as toe angle and camber angle, also can provide theoretical reference for construction design of Automotive Suspension Kinematics Test Bench.
\end{abstract}

\section{Introduction}

Automobile Suspension Kinematics feature is very important to vehicle performance of handling, braking and riding [1]. Center coordinate and geometry attitude of wheel is the core and basic parameters for Suspension Kinematics [2]. Combining space vector geometry calculation and coordinate transform, a new algorithm is designed to calculate feature of Suspension Kinematics. The calculation of the relative coordinates and the displacement of wheel center is by vector operations under the working condition of suspension loading plate move up and down [4]. Other Kinematic parameters also can be calculated by the spatial geometric relationships.

\section{Basic Theory}

In three-dimensional space, we choose right hand spiral direction as the positive direction of rotation of the object in the right hand coordinate system . According to the higher mathematics knowledge and geometry reasoning, the rotation transformation matrix of a point in three dimensional space is a $3 \times 3$ matrix [3][5]:

A points rotation around the positive direction of $X$ axis for angle with $\theta$, the $X$ coordinate values unchanged, $Y$ and $Z$ coordinate values is equivalent to rotation for $\theta$ angle in YOZ plane, then the rotational transformation matrix is:

$$
R_{X}(\theta)=\left(\begin{array}{ccc}
1 & 0 & 0 \\
0 & \cos (\theta) & \sin (\theta) \\
0 & -\sin (\theta) & \cos (\theta)
\end{array}\right)
$$

In the same way, a points rotation around the positive direction of $Y$ or $Z$ axis for angle with $\theta$, Rotational transformation matrix is as follows:

$$
\operatorname{Ry}(\theta)=\left(\begin{array}{ccc}
\cos (\theta) & 0 & -\sin (\theta) \\
0 & 1 & 0 \\
\sin (\theta) & 0 & \cos (\theta)
\end{array}\right), R_{Z}(\theta)=\left(\begin{array}{ccc}
\cos (\theta) & \sin (\theta) & 0 \\
-\sin (\theta) & \cos (\theta) & 0 \\
0 & 0 & 1
\end{array}\right)
$$

So, as shown in figure 1,is a standard to the location and coordinate system of all points, among them, point $O$ is the center of left wheel. Six angle sensors are used to measure the rotation angle of every point relative to the $X, Y$ or $Z$ axis. Rotation angle, respectively is: $\theta 1, \theta 2, \theta 3, \theta 4, \theta 5, \theta 6$ (Corresponding points, respectively is: $A, B, C, D, E, F$, and point $H$ fixed 
connection and can't rotate, $E F, B A$ arm can only rotate limited angle around the $Z$ axis, $E D, D C, C B$ arm can only rotate limited angle around the $X$ axis. $A O$ arm can only rotate limited angle around the $Y$ axis.)
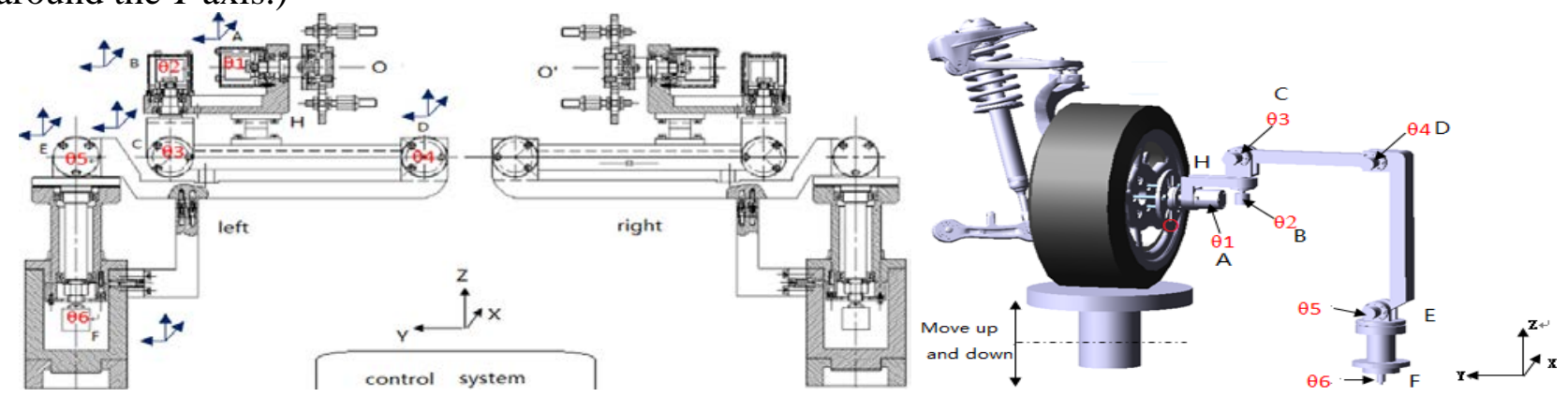

Figure.1. Automobile Suspension Kinematics Test Bench and ADAMS simulation model

Because both sides are completely symmetrical, the algorithm of right is similar to left, now only analysis and research on the algorithm of the left part. After all measuring arms are completely opened, at each measurement point to establish the relative coordinate system respectively.

First, in the point $A$ coordinate system, set $A$ point as the origin of coordinates, so relative to the coordinates of point $A$, the coordinates of point $O$ is:

$O=(0,|\overrightarrow{A O}|, 0)=\overrightarrow{A O}$

Point $O$ rotate $\theta 1$ angle around y axis relative to point $A$ coordinate system and change to point $O 1$, at this time, the coordinates of point $O 1$ is:

$01=\overrightarrow{A O} * R y(\theta 1)=\overrightarrow{A O 1}$

Relative to the coordinates of point $A$, the coordinates of point $B$ is:

$B=(0,-|\overrightarrow{H B}|,|\overrightarrow{A H}|)=\overrightarrow{B A}$

Then, in the triangle $O 1 A B$, Simultaneous equation (3),(4),(5), we can get the coordinates of point $O$ relative to the coordinates of the point $B$ :

$\overrightarrow{B O}=\overrightarrow{B A}+\overrightarrow{A O 1}$

Second, in the point B coordinate system, set $B$ point as the origin of coordinates, after point $O$ rotate $\theta 2$ angle around $Z$ axis relative to point $B$ coordinate system and change to point $O 2$, at this time, the coordinates of point $\mathrm{O} 2$ is:

$02=\overrightarrow{B O} * R Z(\theta 2)=\overrightarrow{B O 2}$

Relative to the coordinates of point $B$, the coordinates of point $C$ is:

$C=(0,0,|\overrightarrow{C B}|)=\overrightarrow{C B}$

Then, in the triangle $O 2 B C$, Simultaneous equation (6),(7),(8), according to vector operation ,we can find:

$$
\overrightarrow{C O}=\overrightarrow{C B}+\overrightarrow{B O 2}
$$

Third, in the point $C$ coordinate system, set $C$ point as the origin of coordinates, after point $O$ rotate $\theta 3$ angle around $X$ axis relative to point $C$ coordinate system and change to point $O 3$, at this time, the coordinates of point $O 3$ is:

$$
03=\overline{C O} * R X(\theta 3)=\overline{C O 3}
$$

Relative to the coordinates of the point $C$, the coordinates of point $D$ is:

$D=(0,|\overrightarrow{D C}|, 0)=\overrightarrow{D C}$

Then, in the triangle $O 3 C D$, Simultaneous equation (9),(10),(11), according to vector operation ,we can find:

$\overrightarrow{D O}=\overrightarrow{D C}+\overrightarrow{C O 3}$

In the same way, using vector operations of triangular, in the coordinate system at the point $D, E$ and $F$, we can find:

$\overrightarrow{E F}=(0,0,-|\overrightarrow{E F}|), \quad \overrightarrow{D E}=(0,0,-|\overrightarrow{D E}|)$

And relative to the coordinates of the point $F$, the coordinates of point $O$ is:

$\overrightarrow{F O}=\left(\left(\overrightarrow{D O} * R_{X}(\theta 4)-\overrightarrow{D E}\right) * R x(\theta 5)+\overrightarrow{F E}\right) * R z(\theta 6)$ 
( $R x(\theta 4), R x(\theta 5)$ is that a point rotate $\theta 4, \theta 5$ degrees around the $\mathrm{X}$ axis . $R z(\theta 6)$ is that a point rotate $\theta 6$ degrees around the $Z$ axis)

At last, Simultaneous equation (1)-(14), that is:

$$
\begin{aligned}
& \overrightarrow{F O}=\left((C)\left(\overrightarrow{D C}+\left(\overrightarrow{C B}+\left(\overrightarrow{B A}+\overrightarrow{A O} * R_{y}(\theta 1)\right) * R_{Z}(\theta 2)\right)\right) * R_{X}(\theta 3)\right) \\
& \left.\left.* R_{X}(\theta 4)-\overline{D E}\right) * R_{X}(\theta 5)+\overline{F E}\right) * R_{z}(\theta 6)
\end{aligned}
$$

In the same way, relative to the coordinates system of point $F$, the coordinates of point $A$ is:$$
\overrightarrow{F A}=\left(\left(C(\overrightarrow{C B}+(-\overrightarrow{B A}) * R Z(\theta 2)) * R_{X}(\theta 3)+(-\overrightarrow{D C})\right) * R_{X}(\theta 4)-\overrightarrow{D E}\right)
$$$$
* R x(\theta 5)+\overrightarrow{F E}) * R z(\theta 6)
$$

\section{Validation and Application of the Algorithm}

Based on the multi-body dynamics related knowledge, the definition of the wheel camber angle is the angle between the wheel plane and the vertical direction of car body, the positive direction is when the top of the wheel is outward. Wheel plane and body level plane have a hand in line, the angle between this line and the longitudinal axis of the body is defined as the wheel toe angle, when the front wheels inward is positive [1] [9].

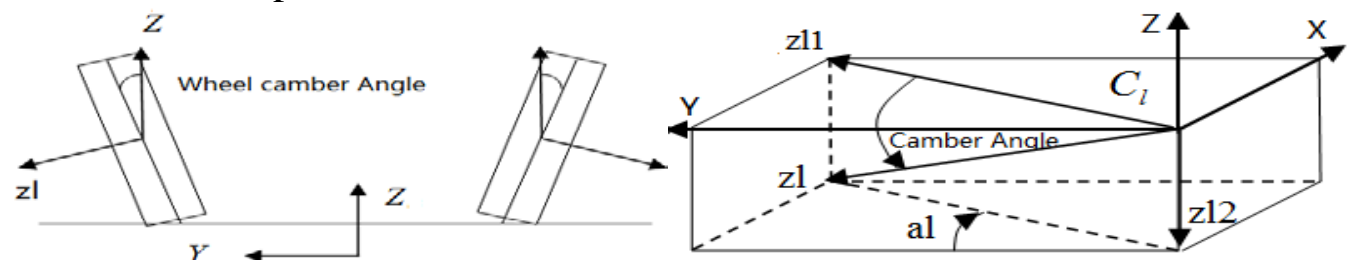

Figure.2. Camber angle and toe angel

As shown in figure 2,set the normal unit vector in the center of the left wheel's rotation plane is $z l$, the unit vector of the vertical direction of the body is $\mathrm{Z}$ axis, the direction of the $z l$ is outside of the body, this vector Can be decomposed into two parts: the vertical direction zl2 and the horizontal direction zl1, the angle between zl1 and Y axis is the toe angle of left wheel, the name is al, the angle between zl1 and zl is the camber angle of left wheel, the name is cl. By the geometric relationships, we can find:

$$
\begin{aligned}
& \overrightarrow{Z l} \cdot \vec{Z}=\cos (90+c l)=-\sin (c l) \\
& \tan \alpha I=\frac{\overrightarrow{z l 1} \cdot \vec{X}}{z l 1 \cdot \vec{Y}}, \overrightarrow{z l 1}=\overrightarrow{z l}-\overrightarrow{z l 2}, \overrightarrow{z l 2} \cdot \vec{X}=0, \overrightarrow{z l 2} \cdot \vec{Y}=0
\end{aligned}
$$

So, Camber angle: $c l=-\sin ^{-1}(\overrightarrow{z l} \cdot \vec{Z})$

$$
\text { Toe angle: } a 1=\tan ^{-1} \frac{\overrightarrow{z l} \cdot \vec{X}}{\overrightarrow{z l} \cdot \vec{Y}}
$$

So, no matter the wheel toe angle or camber angle, we just need to get the vector $z l$.

$$
\overrightarrow{Z I}=\overrightarrow{O A} /|\overrightarrow{O A}|, \overrightarrow{O A}=\overrightarrow{F O}-\overrightarrow{F A}
$$

Simultaneous equation (1)-(19), we can easy get the value of camber angle and toe angle.

In order to validate the correctness of the algorithm, we use the corresponding dynamic simulation model. According to the existing suspension model and measuring system on the basis of CATIA model, set the ADAMS/VIEW model of measurement system, as shown in figure 1 [6][7]. In a certain condition of test work (Set the wheel jumping in the same direction, jumping up and down volume is $70 \mathrm{~mm}$ ). And $O A, A H, H B, B C, C D, D E, E F$ length respectively is: $180,73,163,70,350,480,80$, the unit is $\mathrm{mm}$.

Output variables by the simulation, as follows:

Coordinates of the $O$ relative to $F:(-3.240,690.7,320.2)$, unit: $\mathrm{mm}$.

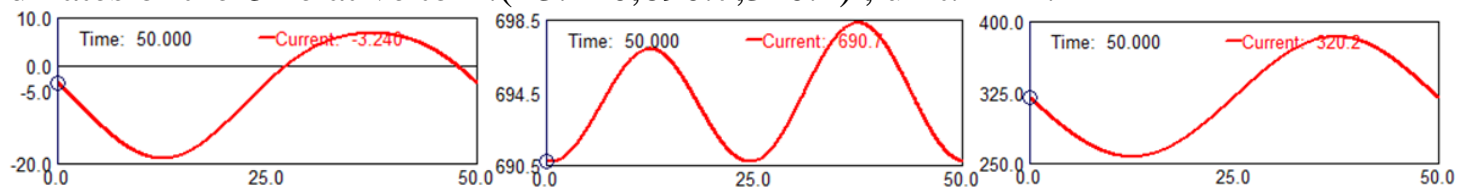

$\theta 1, \theta 2, \theta 3, \theta 4, \theta 5, \theta 6$ are respectively: $-0.2542,-0.5590,2.379,-2.548,0.01615,0.5453$, unit: degree 


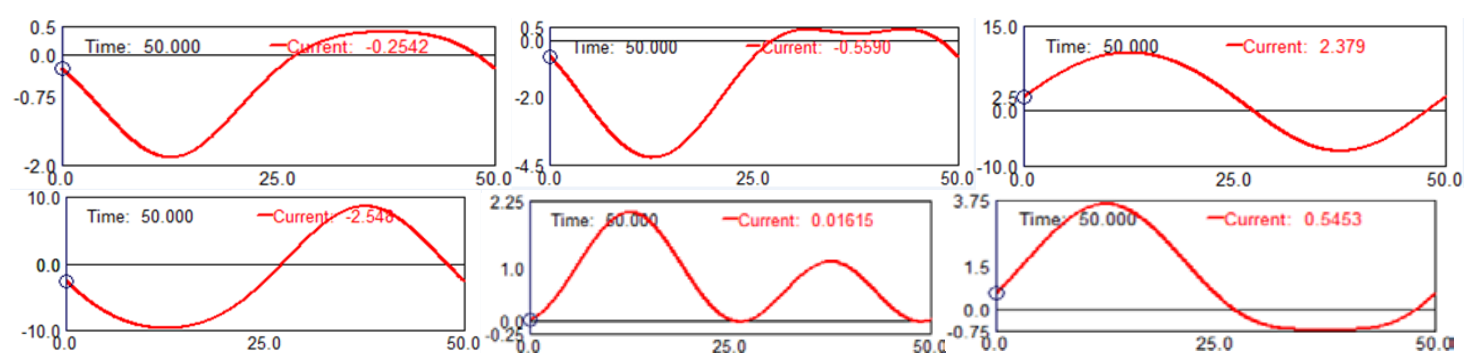

We put the numerical generation into the formula (15), get the coordinates values of point $O$ relative to the coordinate system of point $F$ and compared with the results of Adams output [8]. As shown in figure 3 :

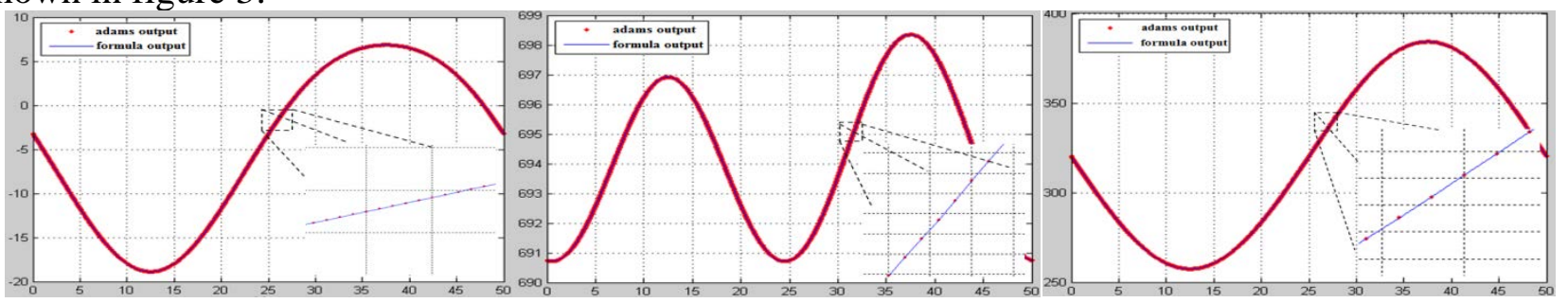

Figure.3. The results of contrast(X, Y, Z)

By comparing the results, we know: the results of this algorithm output and simulation model output are basically identical, so the theory of this algorithm is correct. At the same time, no matter use the simulation model or this algorithm, toe angle and camber angle are got by calculating the coordinates of point. Now, we use the same points and the formula of toe angle and camber angle, so, we get the value of toe angle or camber angle is same by these two methods.

\section{Conclusion}

Based on space coordinate transformation and vector operations, expounded the theory of algorithm about the coordinate of the point of wheel's center, and algorithm was applied to calculate toe angle and camber angle of wheel. Then, based on the ADAMS simulation model to validate the algorithm, the results show that the algorithm is correct and feasible. According to the project's specific needs, the length of each arm of measuring mechanism and the rotation angle can be arbitrarily defined, so space coordinate transform algorithm can provide theoretical reference for construction design of Automotive Suspension Kinematics Test Bench. Algorithm also can be applied to the calculation of main kinematic parameter of others, such as wheel stiffness, suspension stiffness, wheel track and wheelbase, steering gear ratio, etc. Similar engineering test equipment can also learn from this algorithm, such as Three-coordinate Measuring Machine.

\section{References}

[1]Yu Zhisheng. the Theory of Automobile [M]. the Fifth Edition. Beijing: Mechanical Industry Press, 2009.

[2] Isabel Ramirez Ruiz, Dave Fricke, Thomas Stachel.6 DOF Bench Test on a New Active Kinematics Rear Suspension for Functional Development[J].SAE,2012-01-0550.

[3] Ruimei Lian. the Three Dimensional Graphics Geometry Transform and Its Transformation Matrix [J]. Weifang College Journal, 2005.

[4] Xiangdong Sun. the Calculation of 3-D Space Geometric Model [J]. the Development and Application of Computer, 2002.

[5]Sun Jiaguang. Computer Graphics [M]. Beijing: Tsinghua University Press, 1998.

[6]Jinxia Liu, Renxi Hu. From the Introduction to the Master For ADAMS 2012 [M]. Beijing: Mechanical industry press, 2013.

[7] Lijun Qian, Qin Shi. Optimization of Wheel Positioning Parameters of Automotive Front Suspension Based on ADAMS [J]. Information Computing and Applications, 2012.

[8]MathWorks. MATLAB R2011a User’s Guide, 2011.

[9] Dongyoub Baek, Sungmin Cho, Hyunwoo Bang. Wheel Alignment Inspection by 3D Point Cloud Monitoring[J]. Mechanical Science and Technology, 2014 28(4) 1465-1471. 